\title{
Biofilm formation by Streptococcus mutans and its inhibition by green tea extracts
}

\author{
Sara Moataz Zayed', Mohammad Mabrouk Aboulwafa ${ }^{2,3^{*}}$ (D), Abdelgawad Mohamed Hashem ${ }^{4}$ and \\ Sarra Ebrahim Saleh ${ }^{2}$
}

\begin{abstract}
Dental Caries is considered one of the most existing and worldwide common diseases related to the oral cavity affecting both children and adults. Streptococcus mutans is the main cariogenic microorganism involved in the dental caries progression. Natural products such as herbal plants were found to have less side effects and economic value than those of the chemically synthesized antibiofilm agents. This study aimed to isolate Streptococcus mutans from different oral samples taken from saliva and dental plaques specimens to determine their capability for biofilm formation and to evaluate the antibiofilm activity of aqueous and alcoholic green tea extracts. The results revealed that 35, 4 and $1 \%$ of recovered dental plaque isolates exhibited strong, moderate and weak biofilm formation capabilities versus 26, 12 and 2\% for those recovered from saliva. Two green tea extracts (aqueous and alcoholic) were tested for their antibiofilm formation activity against some selected S. mutans isolates. The results showed that the minimum biofilm inhibitory concentrations (MBICs) of the alcoholic and aqueous green tea extracts were in the range of 3.1 to $12.5 \mathrm{mg} /$ $\mathrm{ml}$ and 6.5 to $50 \mathrm{mg} / \mathrm{ml}$, respectively. Accordingly, green tea extracts can be incorporated in various oral preparations for preventing dental caries.
\end{abstract}

Keywords: Streptococcus mutans, Dental caries, Antibiofilm activity, Green tea extracts

\section{Introduction}

Oral biofilm is three-dimensional complex structure of different microorganisms inhabiting the oral cavity; if remained for long time without treatment or intervention, the biofilm can undergo maturation leading to dental caries development. The presence of microorganisms in biofilm structure of exopolysaccharide matrix form enhances their pathogenicity as it improves their resistance to the immune system of the host and different antimicrobial agents (Gurenlian 2015). Dental caries development is associated with biofilm formation affecting large population worldwide. It is believed that the bacteria of species Streptococcus mutans is the primary etiologic agent participating in this serious condition. $S$.

\footnotetext{
*Correspondence: mohammad.aboulwafa@ksiu.edu.eg;

maboulwafa@pharma.asu.edu.eg; maboulwafa@yahoo.com

${ }^{2}$ Microbiology and Immunology Department, Faculty of Pharmacy, Ain

Shams University, Cairo, Egypt

Full list of author information is available at the end of the article
}

mutans plays an essential role in forming multi-dimensional and complex structure on the oral mucosa and tooth enamel (Wang et al. 2020). It acquires some cariogenic properties such as its ability to adhere to solid surfaces, colonize the oral cavity and the ability to survive the acidic condition of the oral cavity (Krzyściak et al. 2014). Also, S. mutans uses the carbohydrate producing acidic metabolites which leads to acidic destruction and demineralization of the tooth enamel removing the mineral materials and thus inducing dental caries. From the virulence factors of $S$. mutans that contribute to oral biofilm development is its ability to produce glucosyltransferase $\mathrm{B}$, an extracellular enzyme which is responsible for glucan formation from sucrose contained in diet. The synthesized glucan causes the adhesion of $S$. mutans to the tooth enamel and other microorganisms to each other that increases protection against mechanical host-clearance forces and different antimicrobial agents (Ito et al. 2020). The essential requirements for biofilm
Springer Open (c) The Author(s) 2021. This article is licensed under a Creative Commons Attribution 4.0 International License, which permits use, sharing, adaptation, distribution and reproduction in any medium or format, as long as you give appropriate credit to the original author(s) and the source, provide a link to the Creative Commons licence, and indicate if changes were made. The images or other third party material in this article are included in the article's Creative Commons licence, unless indicated otherwise in a credit line to the material. If material is not included in the article's Creative Commons licence and your intended use is not permitted by statutory regulation or exceeds the permitted use, you will need to obtain permission directly from the copyright holder. To view a copy of this licence, visit http://creativeco mmons.org/licenses/by/4.0/. 
formation involved in dental caries development related to $S$. mutans are the protein bacterium interactions which involve the sucrose dependent mechanism and the sucrose independent mechanism. The sucrose dependent mechanism mainly depends on glycosyltransferases (GTFB, GTFC and GTFD) produced by S. mutans which are responsible for synthesis of glucan from sucrose. The synthesized sticky nature of glucan provides adhesion of bacteria to each other and cohesion of bacteria to tooth enamel, provides adherence with saliva proteins in pellicle and resistance to clearance by mechanical host forces. While the sucrose independent adhesion is initiated by interaction of salivary agglutinins and $S$. mutans with the surface associated protein P1 (also known as I/II antigen, $\mathrm{SpaP}$ or Pac1. Also, several factors have significant role on biofilm production related to $S$. mutans such as carbohydrate intake and metabolism, aciduricity and acidogenicity. The progression of dental caries is mainly correlated to the diet content of carbohydrate and frequency of their consumption. Sucrose is the most cariogenic carbohydrate as it constitutes the main metabolism for S. mutans. Carbohydrates are mainly taken up by two transporters, phosphoenol pyruvate sugar phosphotransferase system and ATP binding cassette transporters. S. mutans metabolizes carbohydrates to form biofilm and to adhere the microorganisms to each other and to the tooth enamel allowing the pathogen to tolerate different physiological factors such as nutrient availability, aerobic to anaerobic conditions and $\mathrm{pH}$ changes. In addition, S. mutans has the ability to mount and adapt response to low $\mathrm{pH}$ due to ATPase translocating proton contributing in aciduricity, the lower the $\mathrm{pH}$ at which ATPase can function as the metabolic end products builds up, the more competitive the microorganism in biofilm structure. S. mutans is one of the most acidogenic microorganism found in biofilm because it can produce acids from the fermented carbohydrates other than any oral streptococci. In addition, $S$. mutans produces mutacins (bacteriocins) which is important factor in colonization of $S$. mutans in dental biofilm (Banu 2010; Matsumoto-Nakano 2018; Krzyściak et al. 2014).

The currently used chemical antibiofilm agents such as quaternary ammonium salts, iodine compounds and fluoride can lead to various side effects (Rolim et al. 2019; Mehrishi et al. 2020). Also, the overdose and long administration of antimicrobial agents can lead to antimicrobial resistance which is considered a serious health issue nowadays (Razuqi et al. 2012; Fajriani et al. 2020). So, in order to overcome virulence biofilm characters, there has been a shift towards using natural herbal plants that could be used for their possible effects as antibiofilm agents. These natural products have the advantages of being less costly and of lower side effects. Green tea
(Camellia sinensis) has been proposed in this study to be evaluated and tested for its antibiofilm activity. Green tea contains four major flavonoids which are catechins epicatechin (EC), epigallocatechin (EGC), epicatechin gallate (ECG) and epigallocatechin gallate (EGCG) (Fajriani et al. 2020). EGCG plays a significant role in inhibiting biofilm development and progression as it targets the glucosyltransferase enzymes responsible for converting sucrose contained in diet to glucan, the building block of the exopolysaccharide matrix (Hengge 2019).

The aim of this study was to investigate the biofilm formation capability of $S$. mutans isolates collected from dental plaque and saliva specimens and as natural products, two green extracts were used for evaluation of their antibiofilm activity against biofilm former $S$. mutans isolates.

\section{Materials and methods Microorganisms}

A total of 150 isolates were recovered from seventy five dental plaque samples (dp) and seventy five saliva samples (ss) collected from patients having different degrees of dental caries. The samples were obtained from the British University in Egypt (BUE). The clinical specimens of the strongest biofilm producers were deposited at the culture collection of Ain Shams University under the codes of (CCASU-SM 25) and (CCASU-SM 26). The standard bacterial strain Streptococcus mutans (ATCC 25175) was obtained from Faculty of Dentistry, Cairo university. These strains were subcultured routinely on brain heart infusion agar and mitis salivarius medium for maintenance and stored as glycerol stock at $-80{ }^{\circ} \mathrm{C}$ for long term preservation.

\section{Chemicals}

The chemicals used in this study included: glacial acetic acid, glycerol and saline obtained from El-Nasr Chemicals Co., crystal violet powder obtained from SigmaAldrich, methanol obtained from El Gomhouria Co. and chlorhexidine obtained from Arab Drug Company, Cairo, Egypt.

\section{Media and kits}

The used culture media included: brain heart agar, the product of Lab M Ltd., Topley House, Bury, Lanarkshire, United Kingdom; brain heart infusion broth, the product of Oxoid, USA from which two different concentrations of sucrose solution were prepared ( $4 \%$ and $2 \%$ ); mitis salivarius medium, the product of HIMEDIA, India. API 20 Strep kit used for identification of recovered clinical isolates was the product of BioMérieux. 


\section{Recovery and identification of Streptococcus species isolates from collected saliva and dental plaques specimens}

Saliva and dental plaque specimens (75 each) collected from patients suffering from dental caries were used for recovery of Streptococcus species.One loopful from each sample was streaked over plate of mitis salivarius medium and incubated at $37{ }^{\circ} \mathrm{C}$ for $48 \mathrm{~h}$ (Acumedia 2011). The colonies showing the distinct characters of Streptococcus mutans (hard raised, convex, undulate, opaque, pale blue colonies and frosty glass appearance) were picked up and purified on the same medium (Al-mudallal et al. 2008). The purified isolates were identified by commercial biochemical test system API 20 Strep following the manufacturer's instructions. For identification by API 20 Strep, the inoculum was prepared by adding several loopfuls from the pure fresh culture of the test isolate to $2 \mathrm{ml}$ ampoule of API suspension medium to form a dense suspension (4 McFarland). The cupules and tubules of the API Strep were inoculated by the resultant bacterial suspension following the inoculum sizes mentioned by the manufacturer. A drop of mineral oil was added to each cupule for some tests requiring anaerobic conditions. Then the lid was placed on the tray. The inoculated strips were then incubated at $37{ }^{\circ} \mathrm{C}$ and the results were read at the time intervals stated by the manufacturer. For long term preservation, glycerol stock cultures were prepared from single purified colonies of the identified isolates which were then kept at $-80^{\circ}$.

\section{Testing biofilm formation by the recovered isolates Bacterial inoculum preparation}

A pure single colony of each test isolate as well as the standard reference Streptococcus mutans were used for inoculation of $3 \mathrm{ml}$ aliquots contained in wassermann tubes of brain heart infusion broth supplemented with $2 \%$ sucrose. Bacterial cultures were then incubated at $37^{\circ} \mathrm{C}$ for $48 \mathrm{~h}$.

\section{By test tube method (TM)}

The bacterial cultures grown in the wassermann tubes previously prepared were decanted and the tubes were washed gently three times with sterile saline, then dried at room temperature. Three $\mathrm{ml}$ crystal violet solution $(0.1 \%)$ were added to each wassermann tube, then the tubes were left at room temperature for $15 \mathrm{~min}$, followed by decantation of crystal violet solution and three times gentle washing with deionized water. Biofilm formation was detected by the appearance of visible violet colour on the walls and the bottoms of the test tubes. The tubes were photographed and the tested isolates were categorized as zero, absent; 1 , weak; 2 , moderate; 3 , strong biofilm producers (Al-mudallal et al. 2008; Mathur et al. 2006).

\section{By microtiter plate method (MTP)}

The culture prepared before was adjusted to 0.5 McFarland using brain heart infusion broth, then diluted 100 fold in the same medium containing $2 \%$ sucrose. The diluted culture of each test isolate as well as the standard reference strain were distributed in the wells of 96 flat bottom microtiter plate at $200 \mu \mathrm{l}$ per well (3 wells for each isolate). The plates were incubated at $37^{\circ} \mathrm{C}$ for $48 \mathrm{~h}$, then the plates were processed as follows: The bacterial culture was decanted by inversion of microtiter plate and the plates were washed three to four times with sterile saline $(0.9 \%)$. Aliquots $(200 \mu \mathrm{l}$ each) of methanol were added to the washed wells and the plates were left at room temperature for $20 \mathrm{~min}$ followed by decantation of the fixative methanol and leaving the plates to get dry. The plates were stained by adding $200 \mu \mathrm{l}$ of $0.1 \%$ crystal violet to each well and left for $15 \mathrm{~min}$ followed by three times washing with distilled water and the plates were then left at room temperature to get dry while inverted. The adherent cells with their formed biofilms (if any) were resolubilized by adding $200 \mu \mathrm{l}$ of $33 \%$ glacial acetic acid to each well and the OD was then measured at $600 \mathrm{~nm}$ using microplate reader. Each clinical isolate was tested in triplicate, the data was then averaged and standard deviation was estimated. The mean OD value obtained for control wells (containing non inoculated media) was deducted from the average OD values of each test isolate (Kwasny and Opperman 2010). The results obtained were used to classify the test isolates according to their biofilm formation capabilities as shown in Table 1.

\section{Determination of antibiofilm activity of aqueous and alcoholic green tea extracts using microtiter plate assay}

Ten isolates recovered from dental plaque specimens and other 10 recovered from saliva samples were used for testing the antibiofilm activity of aqueous and alcoholic

Table 1 OD (OD600 nm) scale levels used for classification of the tested isolates according to their biofilm formation capabilities (Mohamed et al. 2013)

\begin{tabular}{ll}
\hline OD value & Biofilm formation \\
\hline$O D \leq 2^{*} O D_{c}^{a}$ & Weak biofilm producer \\
$2^{*} O D_{c} \leq O D \leq 4^{*} O D_{c}$ & Moderate biofilm producer \\
$4^{*} O D_{c} \leq O D$ & Strong biofilm producer \\
\hline
\end{tabular}

${ }^{\mathrm{a}} \mathrm{OD}_{\mathrm{c}}$ is $\mathrm{OD}$ of the control wells 
green tea extracts. Each 10 isolates comprised of 2 (weak), 2 (moderate), and 4 (strong) biofilm producers.

\section{Preparation of aqueous green tea extract}

Aqueous green tea extract was prepared by boiling $10 \mathrm{~g}$ grinded green tea leaves (Camellia sinensis) obtained from a herbal medicine store for $5 \mathrm{~min}$ in $100 \mathrm{ml}$ distilled water followed by filtering the cold extract through Whatman filter paper number 1 . The filtrate was then dried at $80{ }^{\circ} \mathrm{C}$ in an incubator and the paste obtained was about $300 \mathrm{mg}$ (Kwasny and Opperman 2010; Faraz et al. 2012). The paste obtained was reconstituted in distilled water to $100 \mathrm{mg} / \mathrm{ml}$.

\section{Preparation of alcoholic green tea extract}

This was carried out similar to the aqueous green tea extract preparation described before except that $50 \mathrm{ml}$ methanol was used instead of 100 of distilled water. The paste obtained was dissolved in distilled water to a concentration of $100 \mathrm{mg} / \mathrm{ml}$ (Kwasny and Opperman 2010; Faraz et al. 2012).

\section{Determination of antibiofilm activity using microtiter plate assay}

The $48 \mathrm{~h}$ brain heart culture of each test clinical isolate was adjusted to $0.5 \mathrm{McF}$ arland using sterile brain heart infusion broth $\left(1.5 \times 10^{8}\right)$. The adjusted suspension for each test isolate was further diluted 100 fold using brain heart infusion broth (represented the isolate inoculum suspension). Aliquots of $100 \mu \mathrm{l}$ of single strength brain heart infusion broth were distributed in the wells of 96 microtiter plate expect the first column of the plate which contained $100 \mu \mathrm{l}$ double strength brain heart infusion broth in each well. Aliquots of $100 \mu \mathrm{l}$ of green tea extract $(100 \mathrm{mg} / \mathrm{ml})$ were transferred to the first well of each row, then two fold serial dilution was carried out up to the tenth columns from which $100 \mu \mathrm{l}$ aliquots were discarded. The last 2 columns (11th and 12th) represent 2 controls, one control was for biofilm formation level of untreated bacterial isolates and the second one represented the biofilm inhibition caused by $0.2 \%$ chlorohexidine. Aliquots $(100 \mu \mathrm{l})$ from the inoculum suspensions of the test isolates were added to wells of three rows assigned to each test isolate. To check sterility of medium, aliquots of $100 \mu \mathrm{l}$ of brain heart infusion broth containing either 2 or $4 \%$ sucrose were added to two separate rows. The inoculated plates were incubated at $37{ }^{\circ} \mathrm{C}$ for $48 \mathrm{~h}$ and the procedures were completed as described before. For each test isolate, the percent inhibition of biofilm formation caused by green tea extract (aqueous or alcoholic) was calculated using the following formula
For the tested agent, the minimum biofilm inhibitory concentration (MBIC) is determined by identifying the lowest concentration of the used agent that inhibited biofilm formation $\geq 80 \%$ (Kwasny and Opperman 2010).

\section{Results \\ Isolation and purification of Streptococcus mutans from saliva and dental plaque specimens}

A total number of 150 bacterial isolates including 75 isolates from saliva and other 75 from dental plaque collected specimens were recovered on mitis salivarius medium. Single colony from each clinical isolate was subcultured on the same medium for further purification and preparation of stock culture for long term preservation at $-80{ }^{\circ} \mathrm{C}$. The saliva sample isolates were given codes from ss1 to ss75 while dental plague isolates were given codes from dp1 to dp75.

\section{Identification of the collected clinical isolates}

Gram staining of the purified clinical isolates were done for preliminary identification, the test isolates showed Gram positive cocci arranged in chains. Isolates were also characterized depending on their colonial shape on mitis salivarius medium. Those suspected to be Streptococcus species showed distinct hard raised, convex, undulate, opaque, pale blue colonies and frosty glass appearance as shown in Fig. 1.

The collected isolates were further checked for their identity using commercial biochemical test system 20600 API 20 strep (BioMérieux) as Streptococcus mutans (100 isolates) and Streptococcus salivarius (50 isolates). The most common characteristic reactions for both Streptococcus species (mutans and salivarius) are represented in Table 2.

\section{Detection of biofilm formation by the collected Streptococcus isolates As determined by tube method (TM)}

The results of tube method (TM) revealed that out of total 150 clinical specimens recovered from oral cavity, 100 isolates were recognized as biofilm producers while the rest of isolates exhibited no biofilm formation capabilities. The presence of violet visible film lining the walls and bottoms of the Wassermann tubes indicates biofilm production and Fig. 2 showed the degree of biofilm formed by some representative isolates. Among

$\left[1-\left(\right.\right.$ average $\mathrm{OD}_{600}$ of treated isolate/average $\mathrm{OD}_{600}$ of untreated isolate $\left.)\right] \times 100$ 


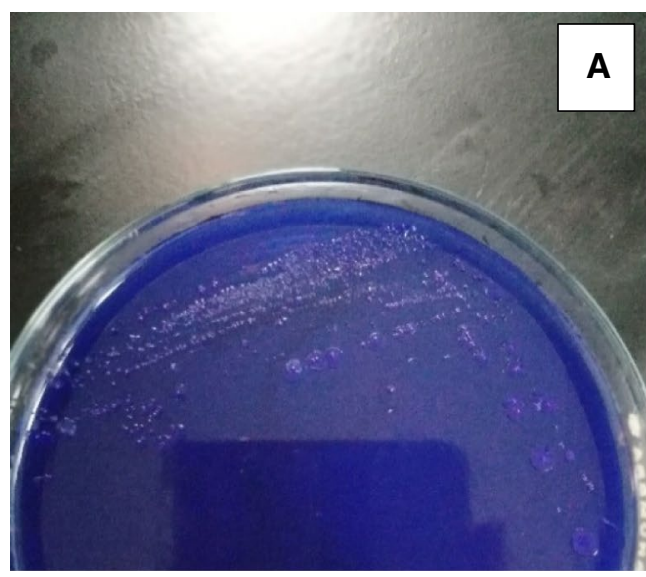

An isolate recovered from a saliva sample

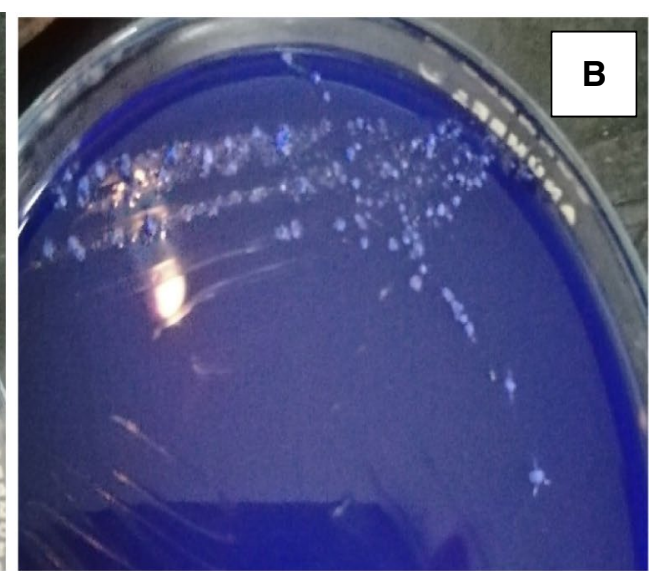

An isolate recovered from a dental plaque sample

Fig. 1 Colonies' appearance of two representative isolates recovered from a saliva (A) and a dental plaque sample (B) on mitis salivarius medium

Table 2 Common biochemical characteristic reactions for $S$. mutans and S. salivarius as determined by API 20 Strep

\begin{tabular}{lll}
\hline Test (Substrate/Reaction/Enzyme) & Result & \\
\cline { 2 - 3 } & S. mutans & S. salivarius \\
\hline VP (Acetoin production) & + & + \\
Hydrolysis (HIPpuric acid) & - & - \\
ESC (B- glucosidase hydrolysis) & + & + \\
PYRA (pyrrolidinyl arylamidase) & - & - \\
aGAL (a-Galactosidase) & + & - \\
BGUR(B-Glucuronidase) & - & - \\
BGAL (B-Galactosidase) & - & - \\
PAL (Alkaline phosphatase) & - & + \\
LAP (Leucine aminopeptidase) & + & + \\
ADH (Arginine dihydrolase) & - & - \\
RIB (D-Ribose) & - & - \\
ARA (L-Arabinose) & - & - \\
MAN (D-Mannitol) & + & - \\
SOR (D-Sorbitol) & + & - \\
LAC (D-Lactose) & + & - \\
TRE (D-Trehalose) & + & + \\
INU (Inulin) & + & - \\
RAF (D- Raffinose) & + & + \\
AMD (Starch) & - & + \\
GLYG (Glycogen) & & - \\
\hline
\end{tabular}

the biofilm producers, 7, 22 and 71 isolates were considered as weak, moderate and strong biofilm producers, respectively as shown in Fig. 3.

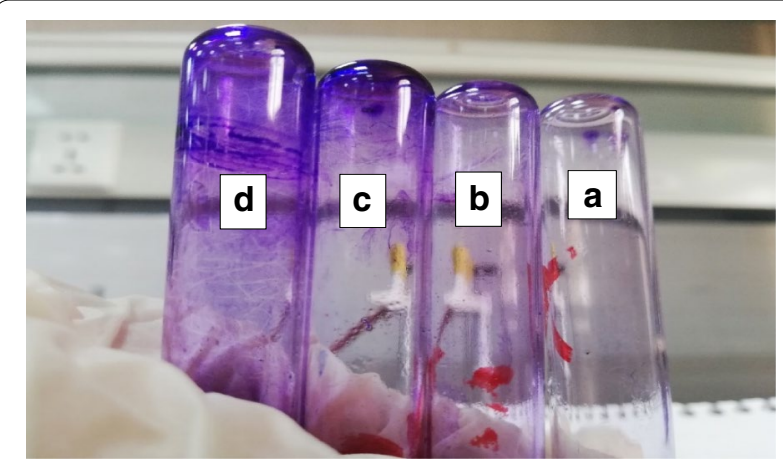

Fig. 2 Violet color developed by some Streptococcus mutans isolates showing different degrees of their formed biofilms as determined by test tube method. (a) non biofilm producer, (b) weak biofilm producer, (c) moderate biofilm producer and (d) strong biofilm producer

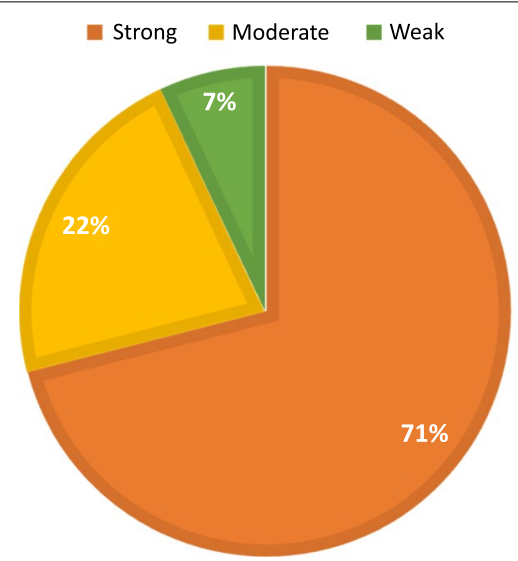

Fig. 3 Relative percentages of S. mutans isolates showing weak, moderate, and strong biofilms 


\section{As determined by microtiter plate method (MTP)}

The results of microtiter plate (MTP) assay showed that out of 100 Streptococcus mutans test isolates only 80 test isolates form biofilm, half of these isolates were recovered from dental plague samples while the rest were recovered from saliva samples. The pattern of biofilm formation capability of Streptococcus mutans test isolates recovered from saliva samples was different from those recovered from dental plaque. Testing forty isolates recovered from each of saliva and dental plaque specimens showed that 26,12 and 2 of saliva isolates were strong, moderate and weak biofilm producers versus 35,4 and 1 for dental plaque isolates (Fig. 4).

The number and codes of isolates showing strong, moderate and weak biofilm production of $S$. mutans recovered from dental plaque and saliva samples as determined by microtiter plate are shown in Table 3.

Some isolates showing different biofilm formation degrees were selected for testing antibiofilm activity of green tea extracts.

\section{Determination of antibiofilm activity of green tea extracts against some selected $S$. mutans isolates}

Aqueous and alcoholic green tea extracts were assayed for their antibiofilm activity using 20 Streptococcus mutans isolates, which included 10 recovered from

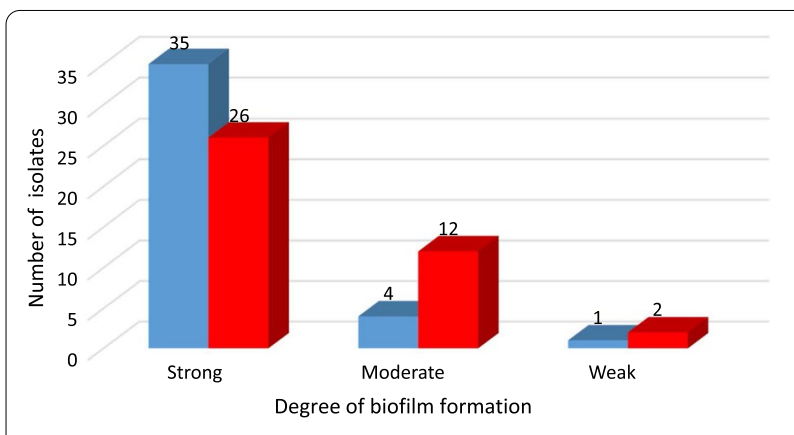

Isolates recovered from dental plaque samples Isolates recovered from saliva samples

Fig. 4 Relative number of isolates showing strong, moderate, and weak biofilm formation of Streptococcus mutans test isolates recovered from dental plaque and saliva samples saliva and other 10 from dental plaque specimens. The selected isolates from saliva samples included 7 strong ( 2 ss (CCASU-SM 25), 5 ss, 13 ss, 14 ss, 24 ss, 30 ss and $40 \mathrm{ss}), 1$ moderate ( $21 \mathrm{ss}), 2$ weak ( $27 \mathrm{ss}, 28 \mathrm{ss}$ ) while the selected isolates from dp samples included 6 strong (dp1, dp16, dp25, dp30 (CCASU-SM26), dp35 and 37dp), 3 moderate (dp 2, dp 6, dp 27), 1 weak (dp 4).The activity was measured by determining minimum biofilm inhibitory concentration (MBIC) for each extract against the test isolates and the results are depicted in Table 4.

\section{Discussion}

Dental caries is one of the most common diseases affecting the oral cavity which is mostly caused by the facultative anaerobic bacteria, Streptococcus mutans. Its ability to form biofilm and to provide adhesion of microorganism to each other and to the tooth enamel, play an important role in developing dental caries which could further lead to serious condition of infective endocarditis. So, preventing this disease was and still one of the most challenging problems in the oral dental practice. Its main pathogenicity is related to its ability to increase resistance against different antimicrobial agents and to be less phagocytized by immune cells (Gurenlian 2015). The adherence and attachment of $S$. mutans to tooth enamel is mainly due to its ability to synthesize glucan, the building block of polysaccharide matrix from sucrose that increases the efficacy of adhesion and attachment. S. mutans possess glucosyltransferase (GTF) enzyme that converts the sucrose to fructose and glucose which is added to growing polymer of glucan forming growing exopolysaccharide matrix. The cariogenic bacteria encodes three genes of glucosyltransferase enzymes (gtfs) (gtfB, gtfC, gtfD). GTFB and GTFC enzymes synthesize water-insoluble glucans which are rich in -1,3-glucosidic linkages while GTFD, produces water-soluble glucans which are rich in -1,6-glucosidic linkages enhancing the coherence of microorganism to each other and adherence to the tooth enamel (Lu et al. 2019; Matsumoto-Nakano 2018). The glucan enhances adhesion of $S$. mutans by forming hydrogen bond to salivary pellicle and other bacteria increasing the biofilm resistance to different

Table 3 Numbers and codes of S. mutans isolates recovered from dental plaque and saliva samples showing strong, moderate and weak biofilm production as determined by microtiter plate

\begin{tabular}{llr}
\hline Biofilm formation degree & \multicolumn{2}{l}{ Numbers and codes () of isolates recovered from: } \\
\cline { 2 - 3 } & Dental plaque samples & Saliva samples \\
\hline Strong & $35(\mathrm{dp} 1,3,5,8,9,10,11,12,13,14,15,16,17,18,19,20,21,22,23,24,25,26,28,29,3$ & $26(\mathrm{ss} 1,2,3,5,6,9,10,11,12,13,14,15,16,18,20,2$ \\
& $0,31,32,33,34,35,37,40,41,45,50)$ & $2,23,24,29,30,32,33,38,40,41,44)$, \\
Moderate & $4(\mathrm{dp} 2,6,7,27)$ & $12(\mathrm{ss} 17,19,21,25,26,31,34,35,36,37,39,42)$ \\
Weak & $1(\mathrm{dp} 4)$ & $2(\mathrm{ss} 27,28)$ \\
\hline
\end{tabular}


Table 4 Antibiofilm activity of alcoholic and aqueous green tea extracts against 20 Streptococcus mutans isolates recovered from dental plaque and saliva specimens (10 isolates each)

\begin{tabular}{|c|c|c|c|}
\hline \multirow{2}{*}{$\begin{array}{l}\text { Biofilm } \\
\text { formation } \\
\text { degree }\end{array}$} & \multirow[t]{2}{*}{ Isolate code } & \multicolumn{2}{|c|}{ MBIC $(\mathrm{mg} / \mathrm{ml})$ of green tea extracts } \\
\hline & & $\begin{array}{l}\text { Aqueous } \\
\text { extract }\end{array}$ & Alcoholic extract \\
\hline \multirow[t]{13}{*}{ Strong } & $\begin{array}{l}\text { ss } 2 \text { (CCASU-SM } \\
25)\end{array}$ & $25 \pm 0.026$ & $6.25 \pm 0.020$ \\
\hline & ss 5 & $25 \pm 0.029$ & $6.25 \pm 0.035$ \\
\hline & ss 13 & $12.5 \pm 0.153$ & $6.25 \pm 0.045$ \\
\hline & ss 14 & $50 \pm 0.1$ & $6.25 \pm 0.050$ \\
\hline & ss 24 & $25 \pm 0.173$ & $12.5 \pm 0.040$ \\
\hline & ss 30 & $50 \pm 0.11$ & $12.5 \pm 0.055$ \\
\hline & ss 40 & $6.25 \pm 0.029$ & $3.125 \pm 0.050$ \\
\hline & $\mathrm{dp} 1$ & $25 \pm 0.1$ & $12.5 \pm 0.032$ \\
\hline & $\mathrm{dp} 16$ & $12.5 \pm 0.098$ & $6.25 \pm 0.045$ \\
\hline & dp 25 & $25 \pm 0.11$ & $6.25 \pm 0.088$ \\
\hline & $\begin{array}{l}\text { dp } 30 \text { (CCASU- } \\
\text { SM 26) }\end{array}$ & $50 \pm 0.091$ & $12.5 \pm 0.02$ \\
\hline & dp 35 & $6.25 \pm 0.161$ & $3.125 \pm 0.25$ \\
\hline & dp 37 & $50 \pm 0.11$ & $12.5 \pm 0.02$ \\
\hline \multirow[t]{4}{*}{ Moderate } & ss 21 & $12.5 \pm 0.068$ & $6.25 \pm 0.09$ \\
\hline & $\mathrm{dp} 2$ & $25 \pm 0.05$ & $12.5 \pm 0.05$ \\
\hline & $\mathrm{dp} 6$ & $25 \pm 0.1$ & $6.25 \pm 0.03$ \\
\hline & $\mathrm{dp} 27$ & $12.5 \pm 0.195$ & $12.5 \pm 0.1$ \\
\hline \multirow[t]{3}{*}{ Weak } & ss 27 & $6.25 \pm 0.147$ & $6.25 \pm 0.07$ \\
\hline & ss 28 & $6.25 \pm 0.053$ & $3.125 \pm 0.04$ \\
\hline & $\mathrm{dp} 4$ & $6.25 \pm 0.06$ & $3.125 \pm 0.11$ \\
\hline
\end{tabular}

* The activity was expressed as minimum biofilm inhibitory concentration (MBIC)

chemotherapeutic agents and the host defense (Banas 2004). Also, another component of S. mutans mediating binding the microorganism to glucan is glucan binding protein (Gbp) composing of 4 types Gbp A, B, C and D. The GbpC and GbpB are associated with the bacterial cell wall of $S$. mutans acting as a specific receptor for glucan which plays a role in microorganism adhesion and biofilm formation (Krzyściak et al. 2014).

In this study, tube and microtiter plate methods were used to detect and quantify biofilm formation of Streptococcus test isolates. A total number of 150 Streptococcus test isolates were tested for their biofilm capability as determined by tube method. About 100 test isolates (66.67\%) were found to be biofilm producers and 50 test isolates (33.34\%) showed no biofilm production. The 100 test isolates showed to be biofilm producers were identified as Streptrococcus mutans while the rest 50 test isolates were recognized as $S$. salivarius (Steiner-Oliveira et al. 2007). According to the results shown by tube method all the 50 tested isolates which did not show any biofilm production activity were identified as $S$. salivarius and this may be due to that $S$. salivarius is a non-biofilm forming bacteria and does not communicate with other microorganisms incorporated in biofilm matrix on the tooth enamel. The biofilm inhibitors produced by $S$. salivarius such as; fructosyltransferase (FTF) and exo-betaD-fructosidase (FruA) which are highly secreted in medium of high concentration of sucrose has a role in biofilm inhibition. As, FruA cleavages sucrose and inhibits the production of glucan which contributes mainly in biofilm development (Steiner-Oliveira et al. 2007; Ogawa et al., 2011). Also, the results of our study were found to be in accordance with another study performed by Pita et al. comparing between biofilm formation between $S$. mutans and $S$. salivarius. Pita et al. stated that among $S$. mutans and S. salivarius in biofilm formation and development, S. salivarius exhibited the lowest capacity to form biofilm compared to S. mutans (Bidossi et al. 2018; Pita et al. 2015).

The test isolates of S. mutans (100) were further screened for their biofilm production by microtiter plate method as this method provides an accurate measurement of biofilm formation by microorganisms. As determined by the microtiter plate, it was observed that the dental plaque samples have the ability to form higher quantities of strong biofilm pattern compared to those of saliva specimens, this is evidenced by the strong biofilm production by 35 isolates recovered from dental plaque compared to only 26 isolates recovered from saliva. Also, 4 test isolates recovered from dental plaque (5\%) showed moderate biofilm production capability versus that of 12 test isolates recovered from saliva specimens (15\%) and 1 test isolate recovered from dental plaque (1.25\%) showed weak biofilm production capability in comparison to 2 test isolates recovered from saliva sample (2.5\%). Comparing the data obtained from samples recovered from dental plaque and saliva samples it was observed that isolates recovered from dental plaque have the ability to form stronger biofilm than those recovered from saliva samples. This may be related to the low concentration of $S$. mutans in saliva samples (Al-mudallal et al. 2008; Banas 2004). The results showed that 35 Streptococcus test isolates recovered from dental plaque (43.75\%) were strong biofilm producers while the same corresponding character degree was exhibited by only 26 Streptococcus test isolates recovered from saliva (32.5\%). Leal and Mickenautsch reported that this can be attributed to that the percentage of $S$. mutans in dental plaque was higher than that of saliva which consequently may be due to the variability of sampling method in saliva and dental plaque specimens and this can affect the pattern of formed biofilm (Leal and Mickenautsch 2010). The prevalence of biofilm production among dental plaque isolates as compared to those isolated from saliva isolates could be due to dental plaque constituted an immobilized surface for 
adherence of microbial community while saliva represents mobilizing viscous solution that could be of low support for biofilm formation. Also, dental plaque represents deposits of debris which act as a substrate for glucan formation by Streptococcus species (Hsu et al. 2010; Motisukiet al. 2005). Saliva contains enzymes such as lysozyme that act adversely on the microbial community (Al-mudallal et al. 2008; Steiner-Oliveira et al. 2007). The antimicrobial mechanism of lysozyme is related to its enzymatic activity in the bacterial peptidoglycan where it hydrolyses $\beta-1,4$ glycosidic bonds between $\mathrm{N}$-acetylglucosamine and $\mathrm{N}$-acetylmuramic acid and because of its cationic and hydrophobic properties, lysozyme has the ability to aggregate oral bacteria affecting their adherence to the tooth enamel, distributing the biofilm structure and enhancing clearance (Hukić et al. 2018).

Different treatment prescriptive in dental caries for biofilm inhibition have been investigated such as chlorhexidine as one of the first antiseptic agents used for dental caries. It has plaque inhibitory activity and blocks the activity of acidic glycoprotein present in saliva, thus decreases the tooth plaque adhesion but it was reported to have serious side effects such as vomiting, diarrhea and tooth discoloration. Quaternary ammonium salts have proposed to be used as antibiofilm agents as the positive charged quaternary ammonium moieties bind to negatively charged bacteria, thus disturb their nature balance. However, these agents have side effects such as coma, convulsions and hypotensive actions which limit their use. Cetylpyridinium chloride has proven to have antibacterial activity by affecting the cell membrane of bacteria decreasing the biofilm development and also polyvinylpyrrolindine inhibits biofilm formation by penetrating the bacterial membrane and inhibiting the metabolism of protein, fatty acid and nucleotides of bacterial cell. Fluoride inhibits enolase enzyme affecting bacterial growth adversely and reduces acid production of $S$. mutans. Long term administration of this agent at high concentration can lead to dental and skeletal fluorosis, thus its use should be reconsidered (Razuqi et al. 2012; Hengge 2019).

The continuous search for alternatives other than the present antibiofilm agents became a required demand because of the high economic cost of the present used synesthetic antibiofilm agents and the possibility of developing serious side effects upon their long term administration (Qiu et al. 2020). The use of herbal products has a low-cost value, least probability of developing major side effects comparable to the conventional antibiofilm agents that are available. So, in this study two extracts from the herbal green tea plant (Camellia sinensis) were checked for their antibiofilm activity (Lu et al. 2019).
Green tea is known to have potential of antibacterial activity. The health-promoting effects of green tea are mainly attributed to its polyphenol contents commonly referred to as catechins. There are four main types of catechins: epigallocatechin-3- gallate (EGCG), epigallocatechin, epicatechin-3- gallate and epicatechin (Faraz et al. 2012). Epigallocatechin-3- gallate (EGCG) exhibits antibiofilm activity mainly by reducing the adherence and attachment of $S$. mutans to the surface of tooth enamel, interfering with bacterial glucosyltransferases (GTF) through reduction of the expression of three genes $(\mathrm{gtfB}$, $g t f C, g t f D)$ that encode these enzymes. Glycosyltransferases enzymes are responsible for converting sucrose to glucan, the building block of the biofilm-associated exopolysaccharide matrix thus inhibiting the biofilm formation (Hengge 2019). Also, another study demonstrated that the green tea may have indirect antibacterial activity by acting on protective saliva components such as secretory immunoglobulins, lysozyme, lactoferrin, oral peroxidases, histatins and mucins (Salama and Alsughier 2019; Taylor et al. 2005). In other recent studies, biofilm formation was suggested to be involved in intercellular cell to cell communication to each other by quorum sensing, EGCG may disturb signaling via autoinducer-1/LuxRdependent or autoinducer-2-dependent quorum sensing systems (Hengge, 2019; Miquel et al. 2016).

The antibiofilm activity of aqueous and alcoholic green tea extracts was evaluated by microtiter plate assay against some selected test isolates of Streptococcus mutans collected and recovered from saliva and dental plaque specimens. The results showed that aqueous and alcoholic green tea extracts have antibiofilm activity against Streptococcus mutans.

The prepared alcoholic green tea extract was observed to have antibiofilm activity at lower concentration than that of aqueous extract. This could be due to its content of more active ingredients, alcoholic extract may have more content of the active constituents epicatechin and epigallocatechin-3- gallate (EGCG) than that of aqueous extract and as it appears from several studies, the antibacterial action of green tea is due to the polyphenolic components (Mageed and Saliem 2015; Blumberg et al. 2015).

It was also observed that alcoholic extract of green tea exhibited antibiofilm activity against the strong biofilm producer isolates at concentrations $3.1,6.3,12.5 \mathrm{mg} / \mathrm{ml}$ and the aqueous extract of green tea exhibited antibiofilm activity at concentrations $6.3,12.5,25$ and $50 \mathrm{mg} / \mathrm{ml}$.

From this study, it can be concluded that the variability in sampling method in dental plaque and saliva samples may affect pattern of biofilm producing bacterial species. Streptococcus mutans species were shown to be biofilm producers in contrast to that of Streptococcus 
salivarius which showed no biofilm formation activity. Green tea can be used for dental caries prevention as it is available and of low economic cost. Both alcoholic and aqueous extracts have antibiofilm activity against the cariogenic bacteria Streptococcus mutans. Thus, these extracts can be used for preparation of antibiofilm dental formulas to be applied for therapeutic purposes or in oral hygiene practice. The alcoholic extract proved to have antibiofilm activity at lower concentrations than that of aqueous extract indicating the extraction of more types and/or amounts of components with antibiofilm activity.

\begin{abstract}
Abbreviations
S. mutans: Streptococcus mutans; S. salivarius: Streptococcus salivarius; MBIC: Minimum biofilm inhibitory concentration; dp: Dental plaque; ss: Saliva sample; EC: Epicatechin; EGC: Epigallocatechin; ECG: Epicatechin gallate; EGCG: Epigallocatechin gallate; OD: Optical density; TM: Test method; MTP: Microtiter plate; GTF: Glucosyltransferase; Gbp: Glucan binding protein; FTF: Fructosyltransferase; FruA: Exo-beta-D-fructosidase; VP: Acetoin production; ESC: BGlucosidase hydrolysis; PYRA: Pyrrolidinyl arylamidase; aGAL: a-Galactosidase; BGUR: B-Glucuronidase; BGAL: B-Galactosidase; PAL: Alkaline phosphatase; LAP: Leucine aminopeptidase; ADH: Arginine dihydrolase; RIB: D-Ribose; ARA: L-Arabinose; MAN: D-Mannitol; SOR: D-Sorbitol; LAC: D-Lactose; TRE: D-Trehalose; INU: Inulin; RAF: D-Raffinose; AMD: Starch; GLYG: Glycogen.
\end{abstract}

\section{Acknowledgements}

The authors acknowledge staff members and professionals at faculty of dentistry, British University in Egypt (BUE) for providing dental plaque and saliva samples that were recovered from patients having different degrees of dental caries. Also, the authors are grateful to staff at Faculty of Dentistry, Cairo university for providing us a copy of the standard bacterial strain Streptococcus mutans (ATCC 25175).

\section{Authors' contributions}

SMZ conducted the practical experiments. SES supervised the lab experiments and shared in writing the manuscript. MMA analyzed the data, shared in writing and revised the manuscript. AMH supervised the work and shared in writing the manuscript. All authors read and approved the final manuscript.

\section{Funding}

No funding was granted for this study.

\section{Availability of data and materials}

The original data and the datasets supporting the conclusions of this current study were included within the article, any required explanation and help is available through the corresponding author.

\section{Declarations}

\section{Ethics approval and consent to participate.}

The research ethics committee for experimental and clinical studies at Faculty of Pharmacy Ain shams university, Cairo, Egypt has approved the experimental studies under the approval serial number (157).

\section{Consent for publication}

Not applicable. All authors approved the manuscript in its final form.

\section{Competing interests}

There are no competing interests among authors.

\section{Author details}

'Medical Sciences Department, Faculty of Dentistry, The British University in Egypt, Cairo, Egypt. ${ }^{2}$ Microbiology and Immunology Department, Faculty of Pharmacy, Ain Shams University, Cairo, Egypt. ${ }^{3}$ Faculty of Pharmacy, King
Salman International University, South Sinai, Ras-Sedr, Egypt. ${ }^{4}$ Microbiology and Immunology Department, Faculty of Pharmacy, The British University in Egypt, Cairo, Egypt.

Received: 2 February 2021 Accepted: 15 May 2021

Published online: 25 May 2021

\section{References}

Acumedia (2011) Mitis Salivarius Agar. 4-5

Al-Mudallal N, Al-Jumaily E, Muhimen N, Al-Shaiban A (2008) Isolation and Identification of mutan'S Streptococci. ANJS 11(3):98-105

Banas A (2004) Virulence properties of Streptococcus mutans. Front Biosci 9(1-3):1267. https://doi.org/10.2741/1305

Banu L (2010) Gene expression in Streptococcus Mutans biofilms. Gene Expression, Arch ZORA. https://doi.org/10.5167/uzh-46739

Bidossi A, De Grandi R, Toscano M, Bottagisio M, De Vecchi E, Gelardi M, Drago L (2018) Probiotics Streptococcus salivarius 24SMB and Streptococcus oralis 89a Interfere with biofilm formation of pathogens of the upper respiratory tract. BMC Infect Dis 18(1):1-11. https://doi.org/10.1186/ s12879-018-3576-9

Blumberg J, Bolling B, Chen C, Xiao H (2015) Review and perspective on the composition and safety of green tea extracts. EJNFS 5(1):1-31. https://doi. org/10.9734/ejnfs/2015/12712

Fajriani, Sartini S, Handayani H, Putri D (2020) The role of green tea extract on inhibiting porphyromonas gingivalis as a major periodontitis pathogen: in vitro study. Sys Rev Pharm 2020;11(8):152-155

Faraz N, Islam Z, Rehman R and Sehrish (2012) Antibiofilm forming activity of naturally occurring compound. J Biomedica, vol. 171-75

Hengge R (2019) Targeting bacterial biofilms by the green tea polyphenol EGCG. J Molecules 24(13):15-17. https://doi.org/10.3390/molecules2 4132403

Hsu K, Osgood R, Cutter G, Childers N (2010) Variability of two plaque sampling methods in quantitation of Streptococcus mutans. J Caries Res 44(2):160-164. https://doi.org/10.1159/000314066

Hukić M, Seljmo D, Ramovic A, Ibrisimovic M, Dogan S, Hukić J, Bojic E (2018) The effect of lysozyme on reducing biofilms by Staphylococcus aureus, Pseudomonas aeruginosa, and Gardnerella vaginalis: an in vitro examination. J Microb Drug Resist 24(4):353-58. https://doi.org/10.1089/mdr. 2016.0303

Ito Y, Ito T, Yamashiro K, Mineshiba F, Hirai K, Omori K, Yamamoto T, Takashiba S (2020) Antimicrobial and antibiofilm effects of abietic acid on cariogenic Streptococcus mutans. J Odontology 108(1):57-65. https://doi.org/10. 1007/s10266-019-00456-0

Gurenlian JR (2015) American dental hygienists' association: the role of dental plaque biofilm in oral health. J Dent Hyg 81(5):1-11

Krzyściak W, Jurczak A, Kościelniak D, Bystrowska B, Skalniak A (2014) The virulence of Streptococcus mutans and the ability to form biofilms. Eur J Clin Microbiol Infect Dis 33(4):499-515. https://doi.org/10.1007/ s10096-013-1993-7

Kwasny S, Opperman T (2010) Static biofilm cultures of gram positive. Curr Protoc Pharmacol. https://doi.org/10.1002/0471141755.ph13a08s50

Leal S, Mickenautsch S (2010) Salivary Streptococcus mutans count and caries outcome-a systematic review. J Minim Interv Dent 3(4):137-147

Lu L, Hu W, Tian Z, Yuan D, Yi G, Zhou Y, Cheng Q, Zhu J, Li M (2019) Developing natural products as potential anti-biofilm agents. J Chin Med 14(1):1-17. https://doi.org/10.1186/s13020-019-0232-2

Mageed M, Saif S (2015) Antibacterial effects of green tea extracts on Aggregatibacter actinomycetemcomitans : In vitro study. JBCD 27(3):102-108. https://doi.org/10.12816/0015043

Mathur T, Singhal S, Khan S, Upadhyay FT, Rattan A (2006) Detection of biofilm formation among the clinical isolates of Staphylococci: an evaluation of three different screening methods. Indian J Med Microbiol 24(1):25-29

Matsumoto-Nakano M (2018) Role of Streptococcus mutans surface proteins for biofilm formation. Jpn Dent Sci Rev 54(1):22-29. https://doi.org/10. 1016/j.jdsr.2017.08.002

Mehrishi P, Agarwal P, Broor S, Sharma A (2020) Antibacterial and Antibiofilm properties of medicinal plant extracts against multi drug resistant Staphylococcus species and non fermenter bacteria. J Pure Appl Microbiol 14(1):403-413. https://doi.org/10.22207/JPAM.14.1.42 
Miquel S, Lagrafeuille R, Souweine B, Forestier C (2016) Anti-biofilm activity as a health issue. Front Microbiol. https://doi.org/10.3389/fmicb.2016.00592

Mohamed A, Rajaa A, Khalid Z, Fouad M, Naima R (2013) Comparison of three methods for the detection of biofilm formation by clinical isolates of Staphylococcus aureus isolated in casablanca. IJSR 5(10):2319-7064. https://doi.org/10.21275/ART20162319

Motisuki C, Lima L, Spolidorio D, Santos-Pinto L (2005) Influence of sample type and collection method on Streptococcus mutans and Lactobacillus Spp. counts in the oral cavity. Arch Oral Bio 50(3):341-45. https://doi.org/ 10.1016/j.archoralbio.2004.08.007

Ogawa A, Furukawa S, Fujita S, Mitobe J, Kawarai T, Narisawa N, Sekizuka T, Kuroda M, Ochiai K, Ogihara H, Kosono S, Yoneda S, Watanabe H, Morinaga Y, Uematsu H, Senpuku H (2011) Inhibition of Streptococcus mutans biofilm formation by Streptococcus salivarius FruA. Appl Envivon Microbiol 77(5):1572-1580. https://doi.org/10.1128/AEM.02066-10

Pita P, Rodrigues J, Ota-Tsuzuki C, Miato T, Zenobio E, Giro G, Figueiredo L, Gonçalves C, Gehrke S, Cassoni A, Shibli J (2015) Oral Streptococci biofilm formation on different implant surface topographies. Biomed Res Int. https://doi.org/10.1155/2015/159625

Qiu W, Zhou Y, Li Z, Huang T, Xiao Y, Cheng L, Peng X, Zhang L, Ren B (2020) Application of antibiotics/antimicrobial agents on dental caries. Biomed Res Int. https://doi.org/10.1155/2020/5658212

Razuqi N, Dhahir S, Ascar E (2012) Biological effect of aqueous \& alcoholic extracts of green tea leaves of some pathogenic bacteria in vitro. J Kufa for Chem 10(4):5-16
Rolim W, Lamilla C, Pieretti J, Diaz M, Tortella G, Diez M, Barrientos L, Seabra A, Rubilar $O$ (2019) Comparison of antibacterial and antibiofilm activities of biologically synthesized silver nanoparticles against several bacterial strains of medical interest. J Energ Ecol Environ 4(4):143-159. https://doi. org/10.1007/s40974-019-00123-8

Salama M, Alsughier Z (2019) Effect of green tea extract mouthwash on salivary Streptococcus mutans counts in a group of preschool children: an in vivo study. Int J Clin Pediatr Dent 12(2):133-138. https://doi.org/10. 5005/jp-journals-10005-1610

Steiner-Oliveira C, Maciel F, Rodrigues L, Napimoga M, Pimenta L, Höfling J, Gonçalves R (2007) An in vitro microbial model for producing caries-like lesions on enamel. Braz J Oral Sci 6(22):1392-1396

Taylor P, Hamilton-Miller J, Stapleton P (2005) Antimicrobial properties of green tea catechins. Food Sci Technol Bull 2(7):71-81. https://doi.org/10.1616/ 1476-2137.14184

Wang Y, Shen X, Ma S, Guo Q, Zhang W, Cheng L, Ding L, Xu Z, Jianga J, Gao $L$ (2020) Oral biofilm elimination by combining iron-based nanozymes and hydrogen peroxide-producing bacteria. Biomater Sci 8(9):2447-2458 https://doi.org/10.1039/c9bm01889a

\section{Publisher's Note}

Springer Nature remains neutral with regard to jurisdictional claims in published maps and institutional affiliations.

\section{Submit your manuscript to a SpringerOpen ${ }^{\circ}$ journal and benefit from:}

- Convenient online submission

- Rigorous peer review

- Open access: articles freely available online

- High visibility within the field

- Retaining the copyright to your article

Submit your next manuscript at springeropen.com 